\title{
High-resolution intravascular magnetic resonance imaging of the coronary artery wall at 3.0 Tesla: toward evaluation of atherosclerotic plaque vulnerability
}

\author{
Yanfeng Meng ${ }^{1}$, Zhiguang $\mathrm{Mo}^{2,3}$, Jinying $\mathrm{Hao}^{1}$, Yueyou Peng ${ }^{1}$, Hui Yan ${ }^{1}$, Jingbo Mu${ }^{4}$, Dengfeng $\mathrm{Ma}^{4}$, \\ Xiaoliang Zhang ${ }^{5}$, Ye $\mathrm{Li}^{2,3}$ \\ ${ }^{1}$ Department of MRI, Taiyuan Central Hospital of Shanxi Medical University, Taiyuan, China; ${ }^{2}$ Paul C. Lauterbur Research Center for Biomedical \\ Imaging, Shenzhen Institute of Advanced Technology, Chinese Academy of Sciences, Shenzhen, China; ${ }^{3}$ The Key Laboratory for Magnetic \\ Resonance and Multimodality Imaging of Guangdong Province, Shenzhen, China; ${ }^{4}$ Department of Cardiology, Taiyuan Central Hospital of Shanxi \\ Medical University, Taiyuan, China; ${ }^{5}$ Department of Biomedical Engineering, State University of New York at Buffalo, NY, USA
}

Contributions: (I) Conception and design: Y Meng, Z Mo, Y Li; (II) Administrative support: Y Li, Y Meng; (III) Provision of study materials or patients: None; (IV) Collection and assembly of data: Z Mo, J Hao, Y Peng, H Yan, J Mu, D Ma; (V) Data analysis and interpretation: Y Meng, Z Mo, X Zhang; (VI) Manuscript writing: All authors; (VII) Final approval of manuscript: All authors.

Correspondence to: Ye Li, PhD; Zhiguang Mo. Paul C. Lauterbur Research Center for Biomedical Imaging, Shenzhen Institute of Advanced Technology, Chinese Academy of Sciences, and the Key Laboratory for Magnetic Resonance and Multimodality Imaging of Guangdong Province, Shenzhen, 518055, China. Email: liye1@siat.ac.cn; zg.mo@siat.ac.cn.

Background: To validate the feasibility of generating high-resolution intravascular 3.0 Tesla (T) magnetic resonance imaging of the coronary artery wall to further plaque imaging.

Methods: A receive-only 0.014-inch diameter magnetic resonance imaging guidewire (MRIG) was manufactured for intravascular imaging within a phantom experiment and the coronary artery wall of the swine. For coronary artery wall imaging, both high-resolution images and conventional resolution images were acquired. A 16-channel commercial surface coil for magnetic resonance imaging was employed for the control group.

Results: For the phantom experiment, the MRIG showed a higher signal-to-noise ratio than the surface coil. The peak signal-to-noise ratio of the MRIG and the surface coil-generated imaging were 213.6 and 19.8, respectively. The signal-to-noise ratio decreased rapidly as the distance from the MRIG increased. For the coronary artery wall experiment, the vessel wall imaging by the MRIG could be identified clearly, whereas the vessel wall imaging by the surface coil was blurred. The average signal-to-noise ratio of the artery wall was $21.1 \pm 5.40$ by the MRIG compared to $8.4 \pm 2.19$ by the surface coil, where the resolution was set at $0.2 \mathrm{~mm} \times 0.2 \mathrm{~mm} \times 2 \mathrm{~mm}$. As expected, the high-resolution sequence clearly showed more details than the conventional resolution sequence set at $0.7 \mathrm{~mm} \times 0.7 \mathrm{~mm} \times 2.0 \mathrm{~mm}$. Histological examination showed no evidence of mechanical injuries in the target vessel walls.

Conclusions: The study validated the feasibility of generating magnetic resonance imaging (MRI) at $0.2 \mathrm{~mm} \times 0.2 \mathrm{~mm} \times 2 \mathrm{~mm}$ for the coronary artery wall using a 0.014 inch MRIG.

Keywords: Intravascular MRI; magnetic resonance imaging guidewire (MRIG); artery wall imaging; highresolution

Submitted Mar 16, 2021. Accepted for publication Jul 05, 2021.

doi: $10.21037 /$ qims-21-286

View this article at: https://dx.doi.org/10.21037/qims-21-286 


\section{Introduction}

Atherosclerotic cardiovascular disease is the leading cause of death worldwide. Although tremendous efforts have been made to prevent, diagnose, and treat the disease over the past few decades, mortality continues to increase $(1,2)$. Atherosclerosis primarily begins when plasma cholesterol levels become elevated. This scenario, among other factors, leads to the formation of atherosclerotic plaques (3). Plaque in the artery wall narrows the artery lumen and may result in myocardial infarction or cerebral stroke. Studies have demonstrated that most acute infarctions are correlated with vulnerable plaques (4). Vulnerable plaques are characterized by luminal surface ulceration, intraplaque hemorrhage, a fissured fibrous cap, and lipidrich necrotic core, neovascularization, and inflammation, plaque remodeling, and increased size (5). Rupture of a vulnerable plaque leads to platelet accumulation and artery occlusion, resulting in organ infarction. Magnetic resonance imaging (MRI) is useful for detecting carotid artery plaques and their components, and the imaging modality has helped prevent cerebral infarction (6-10).

In recent years, deep coronary artery wall MRI has been introduced for detecting coronary artery plaques (11). Due to the limitations of the current MRI technique, a voxel size of $0.7 \mathrm{~mm} \times 0.7 \mathrm{~mm} \times 2 \mathrm{~mm}$ is usually used in human coronary artery wall imaging at 3.0 Tesla $(\mathrm{T})(12,13)$. However, the voxel size is larger than the artery wall, which has a thickness of approximately $0.2-0.3 \mathrm{~mm}$ (14). Therefore, the vulnerability of the plaque is difficult to determine directly.

Performing MRI on deep arteries can provide lowresolution images, which presents a challenge in plaque imaging. An intravascular MRI technique was developed, which involved the placement of a magnetic resonance (MR) radio frequency $(\mathrm{RF})$ coil into the target vessel to generate intravascular high-resolution wall imaging to address this challenge $(15,16)$. Due to the limited space in vessels, the intravascular RF coil design and the miniature coaxial transmission line coils $(17,18)$, including loopless MR RF antenna, were advantageous because they were able to fit into smaller vessels and were more easily manipulated (19). The loopless MR antenna, also known as the MRIG, added additional functionality by acting as a conventional guidewire for MR-guided intravascular intervention, such as balloon angioplasty (20). The MRIG was further refined to 0.014 inches (about $0.35 \mathrm{~mm}$ ) in diameter and used on 1.5 T MRI for coronary artery imaging $(21,22)$.

Studies have shown that intravascular imaging can acquire high-resolution vascular images of $0.08 \mathrm{~mm}$ in $e x$ vivo tissues with a $2.2 \mathrm{~mm}$ loopless antenna detector (23). Given the improved sensitivity, the higher magnetic field strength is expected to be beneficial in high-resolution image acquisition (24-30). To the best of our knowledge, no reports exist on the feasibility of high-resolution imaging with an MRIG as thin as 0.014 inches at 3.0 T MRI.

This study aimed to validate the feasibility of generating a high-resolution intravascular 3.0 T MRI of the coronary artery wall for further plaque imaging.

\section{Methods}

\section{Devices}

In this study, a $3.0 \mathrm{~T}$ "receive only" MRIG was manufactured from a 0.014 inch diameter coaxial cable (UL1005, Sumitomo Electric Inc., Osaka, Japan) to fit inside the coronary artery. The coaxial cable conductors were made of Sn-Cu alloy (Sn: $\left.8.8 \times 10^{6} \mathrm{~S} / \mathrm{m}, \mathrm{Cu}: 5.8 \times 10^{7} \mathrm{~S} / \mathrm{m}\right)$. We used a soft coaxial cable as the material of the MRIG, as it had a higher conductivity compared to the Nitinol-based MRIG (Nitinol: $1 \times 10^{6} \mathrm{~S} / \mathrm{m}$ ) (31) and was safer for coronary artery intervention. The inner conductor with an insulation layer was extended $10 \mathrm{~cm}$ beyond the outer conductor shield, and the total length of the MRIG was longer than $40 \mathrm{~cm}$. The insulation layer broadened the longitudinal region over which images could be acquired (32) and improved the strength of the inner conductors.

The proximal end of the coaxial cable was connected to a 3.0 T MR scanner (GE Discovery 750W, GE Healthcare, Best, USA) through a matching/tuning circuit (Figure 1A). Regarding the circuit, the S11 of the MRIG (Figure $1 B$ ) was adjusted to below -20 decibel $(\mathrm{dB})$ at the operating frequency of 127.7 megahertz (MHz). As shown in Figure $1 A$, the capacitances $\mathrm{C} 2$ and $\mathrm{C} 3$ at 13 picofarads $(\mathrm{pF})$, and the inductors L1 and L2 at 120 nanohenry $(\mathrm{nH})$ formed a parallel resonant circuit. The bias voltage was transmitted via the coaxial cable, and the switching diodes D1 and D2 were driven by the bias voltage. When the switches were on, the 2 detuning circuits showed high impedance. It likely protected the scanner from unexpected high voltage surges. By adjusting the capacitances of $\mathrm{C} 4, \mathrm{C} 5$, and $\mathrm{C} 6$, a wide range of frequencies covering the operating frequency was achieved (Figure 2). We set the capacitance of C1 to 1 microfarad $(\mu \mathrm{F})$ to isolate the direct current (DC) signal. In addition, the inductors L3, L4, and L5 at $2.7 \mu \mathrm{H}$ were used to isolate the alternating current (AC) signal. 
A

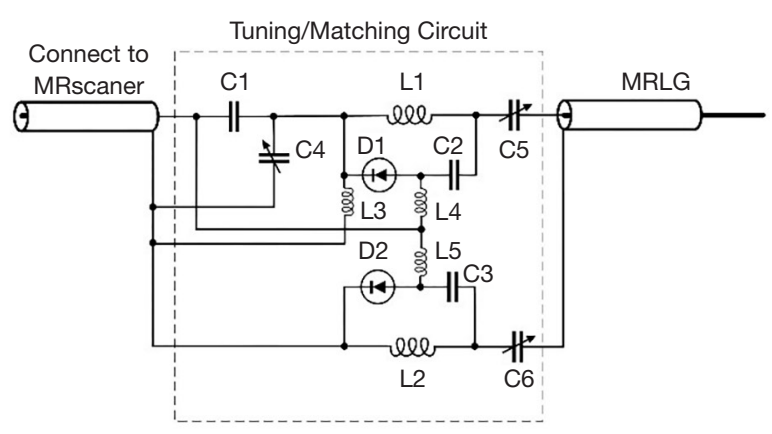

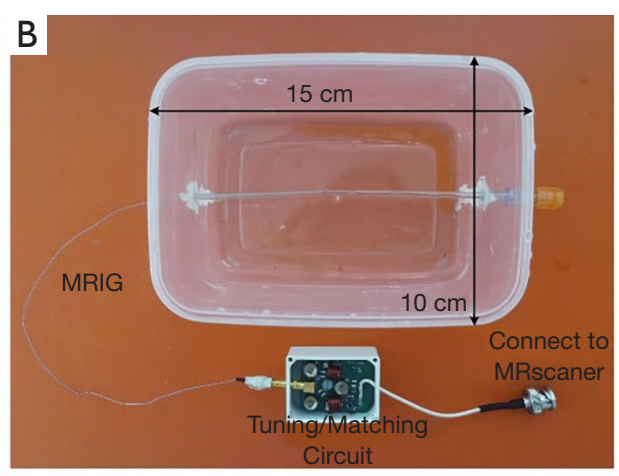

Figure 1 Design of the 0.014 inch MRIG. The MRIG was connected to a coaxial cable through a tuning/matching Circuit (A) and was placed in the plastic tube "vessel" of the box $(150 \mathrm{~mm} \times 100 \mathrm{~mm} \times 90 \mathrm{~mm}$ ) filled with $0.9 \%$ normal saline (B). MRIG, magnetic resonance imaging guidewire.
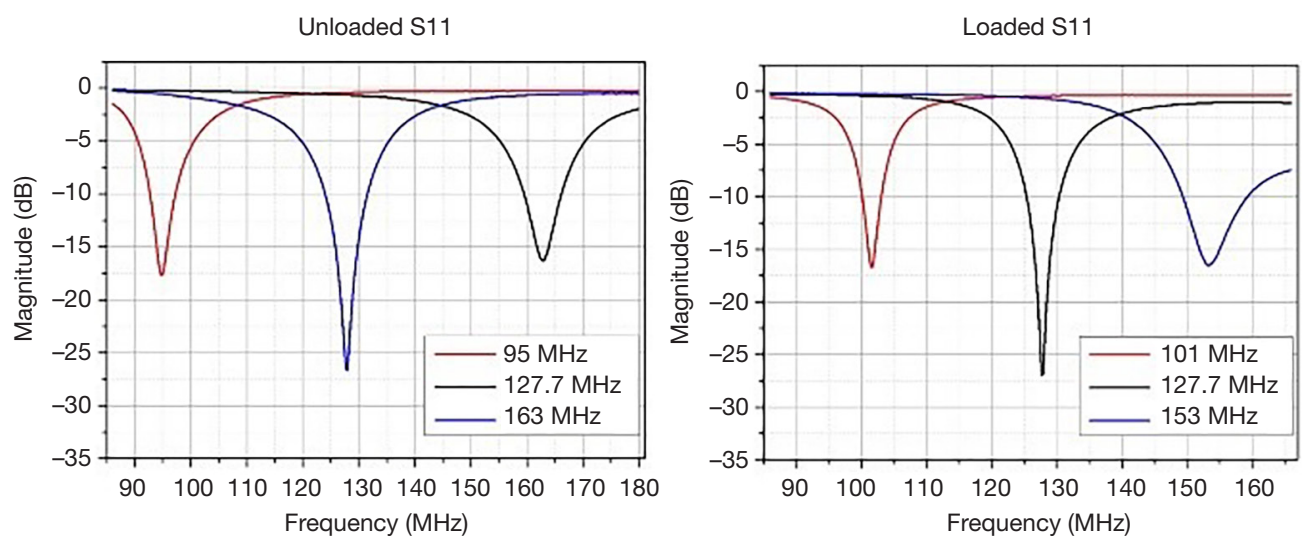

Figure 2 The frequency range of S11 was measured when the MRIG was loaded and unloaded. By adjusting the tuning and matching circuit, the resonance frequency of unloaded S11 ranged from 95 to $163 \mathrm{MHz}$, and the resonance frequency of loaded S11 ranged from 101 to $153 \mathrm{MHz}$, both of which covered the operating frequency of $127.7 \mathrm{MHz}$. The unloaded S11 was obtained by exposing the MRIG to air, and the loaded S11 was obtained by inserting the MRIG into the phantom. MRIG, magnetic resonance imaging guidewire.

\section{In vitro study}

To test the functionality of the MRIG, in vitro experiments were performed. We constructed a plastic box containing a plastic tube inside to mimic the vessel, and filled the plastic tube with $0.9 \%$ normal saline (Figure 3). The outside diameter of the tube measured approximately $3 \mathrm{~mm}$, and the thickness was $0.5 \mathrm{~mm}$. The plastic box and the tube were both made of polyethylene, through which no MR signal could be generated. We placed the MRIG into the plastic tube "vessel". Axial T2-weighted MRI of the plastic tube "vessel" was then acquired using a fast spin-echo (FSE) sequence where repetition time $(\mathrm{TR})=3,000 \mathrm{~ms}$, echo time $(\mathrm{TE})=68 \mathrm{~ms}$, echo train length $=6$, field of view $(\mathrm{FOV})=100 \mathrm{~mm}$ $\times 100 \mathrm{~mm}$, matrix $=512 \times 512$, slice thickness $=2 \mathrm{~mm}$, and number of averages $=4$. With the same MR parameters and position, we acquired phantom imaging with a commercial 16-channel surface coil placed approximately $5 \mathrm{~cm}$ superior to the plastic tube.

\section{Ex vivo study}

For the ex vivo study, we placed a fresh adult swine heart into a plastic box filled with $0.9 \%$ normal saline. After placing the MRIG into the left anterior descending (LAD) branch of the coronary artery, an MRI of the coronary 

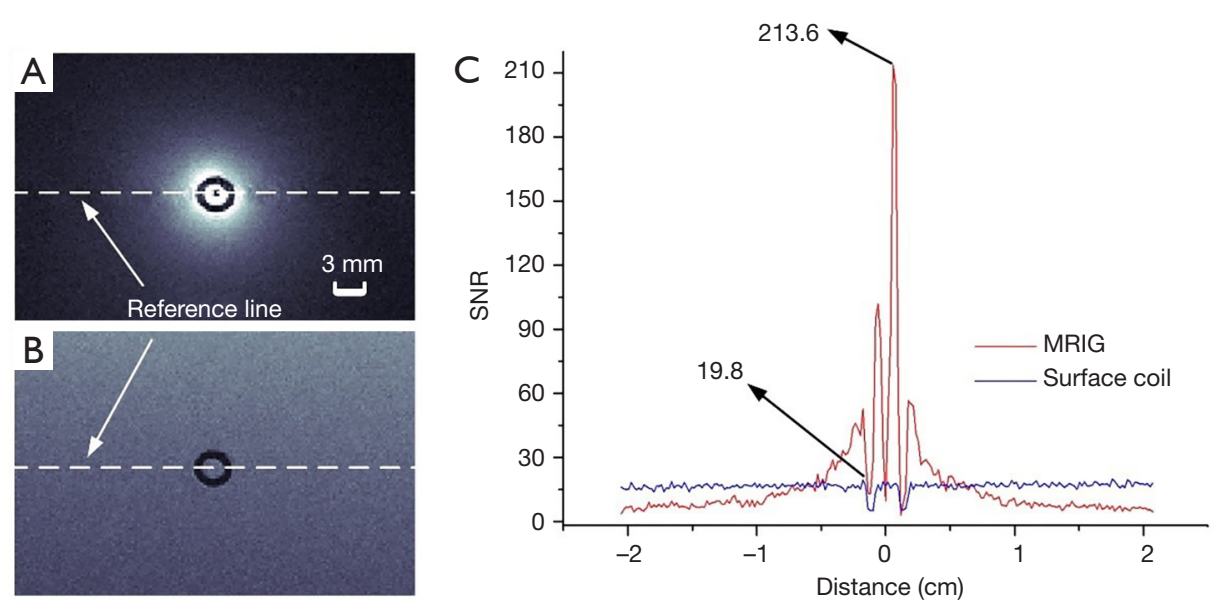

Figure 3 Phantom study with a plastic tube that mimicked a vessel. Axial T2-weighted MRIs with the MRIG (A) compared to the commercial 16-channel surface coil (B). The SNR was compared with two detectors on the reference line, in a space where the plastic tube "vessel" and MRIG showed no signal (C). MRIG, magnetic resonance imaging guidewire.

artery wall was acquired. Axial T2-weighted imaging of the LAD branch was performed using a FSE sequence with TR $=3,000 \mathrm{~ms}, \mathrm{TE}=68 \mathrm{~ms}$, echo train length $=6, \mathrm{FOV}=100 \mathrm{~mm}$ $\times 100 \mathrm{~mm}$, matrix $=512 \times 512$, slice thickness $=2 \mathrm{~mm}$, and number of averages $=4$. Maintaining the same position, we adjusted the FOV and matrix size to $110 \mathrm{~mm} \times 110 \mathrm{~mm}$ and $160 \times 160$, respectively. We then acquired high-resolution and conventional resolution images of the same LAD branch of the coronary artery. The commercial surface coil was placed about $5 \mathrm{~cm}$ superior to the LAD branch to acquire wall imaging using the same settings. The experiments were repeated 4 times, and images were acquired from 4 different swine hearts using the same guidewires.

\section{Data analysis}

After obtaining all the images, the noise was acquired by maintaining all conditions and manually setting the RF gain to 0 . The signal-to-noise (SNR) of each voxel was calculated using the following formula (33):

$$
S N R=\frac{S^{T} S}{\sqrt{S^{T} \psi S}}
$$

where $S$ is the signal intensity of each channel and $\psi$ is the noise resistance. A reference line was drawn through the tube center to demonstrate the spatial SNR distribution visually in the phantom study, and the value that varied with distance from the center was plotted.

In the ex vivo study, the highest achievable SNR was measured by placing the region of interest (ROI) at the brightest part of the coronary artery walls. To calculate the average SNR of the swine arterial wall $\left(S_{w}\right)$, we measured the average SNR $\left(S_{t}\right)$ of the total area and the average SNR $\left(S_{l}\right)$ of the vessel lumen. The $S_{w}$ was calculated using the following formula (34):

$$
S_{\mathrm{w}}=\frac{\left(S_{t} \times A_{t}\right)-\left(S_{l} \times A_{l}\right)}{A_{t}-A_{l}}
$$

Where $A_{t}$ is the total vessel area, and $A_{l}$ is the vessel lumen area.

\section{Histology}

After achieving MRI, the coronary artery segments were harvested and cryosectioned at $5 \mu \mathrm{m}$. We then performed histological examinations with hematoxylin and eosin (HE) staining to detect the integrity of the artery wall.

\section{Results}

In the in vitro experiments, both the MRIG and commercial surface coil functioned well as receiver coils when used with the 3.0 T MR scanner. In both images, the plastic tube "vessel" was able to be identified at the resolution of $0.2 \mathrm{~mm}$ $\times 0.2 \mathrm{~mm} \times 2 \mathrm{~mm}$. The vessel wall imaging by the MRIG could be clearly identified, whereas the plastic tube "vessel" imaged by the surface coil was blurred (Figure $3 A, B$ ). As shown in Figure 3C, a series of SNR values along 

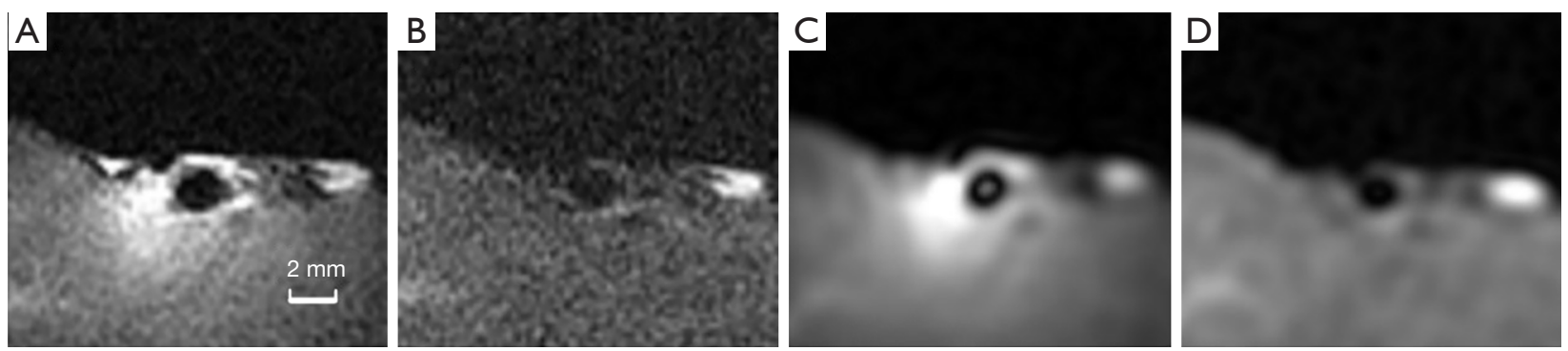

Figure 4 Cross-sectional view of the MRIs of the left LAD branch of the coronary artery of a swine. High-resolution images (A, B) obtained at $0.2 \mathrm{~mm} \times 0.2 \mathrm{~mm} \times 2 \mathrm{~mm}$ and conventional resolution images $(\mathrm{C}, \mathrm{D})$ obtained at $0.7 \mathrm{~mm} \times 0.7 \mathrm{~mm} \times 2 \mathrm{~mm}$ were achieved using the MRIG (A, C) and surface coil (B, D), respectively. LAD, left anterior descending; MRIG, magnetic resonance imaging guidewire.

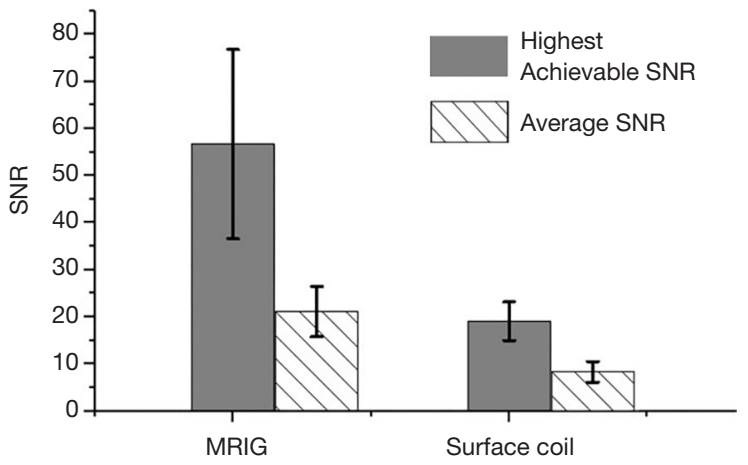

Figure 5 Measurements of the average SNR (Sw) of the LAD branch and the highest achievable SNR (maximum value in SNR map) showing a higher SNR obtained using the MRIG than that which was obtained using the surface coil. The highest achievable SNR of the artery wall was $56.7 \pm 20.1$ compared with the SNR of $19.1 \pm 4.1$ that was obtained using the surface coil. The average SNR obtained using the MRIG was $21.1 \pm 5.40$, compared to $8.4 \pm 2.19$ obtained using the surface coil, where the resolution was set to $0.2 \mathrm{~mm} \times 0.2 \mathrm{~mm} \times 2 \mathrm{~mm}$. SNR, signal-to-noise; LAD, left anterior descending; MRIG, magnetic resonance imaging guidewire.

the reference line through the plastic tube "vessel" was achieved. The peak SNR of the MRIG and the surface coilgenerated imaging was 213.6 and 19.8 , respectively. The signal decreased rapidly as the distance from the MRIG increased. The calculated curve indicated that the SNR was higher than the surface coil in the range of $\pm 0.5 \mathrm{~cm}$ near the intersection of the inner conductor and the outer conductor of the MRIG.

In the ex vivo experiments, LAD branch wall imaging was acquired using the MRIG and the surface coil (Figure 4).
As shown in the images, the MRIG-acquired LAD branch wall was able to be identified at the resolution of $0.2 \mathrm{~mm}$ $\times 0.2 \mathrm{~mm} \times 2 \mathrm{~mm}$. The surface coil-acquired LAD branch wall was blurred. Compared to the high-resolution images, the conventional resolution images of $0.7 \mathrm{~mm} \times 0.7 \mathrm{~mm} \times$ $2 \mathrm{~mm}$ lacked the detail of the high-resolution images. The highest achievable SNR of the artery wall was $56.7 \pm 20.1$ compared to the SNR of $19.1 \pm 4.1$ that was obtained by the surface coil. The average SNR $\left(S_{w}\right)$ of the artery wall obtained by the MRIG was $21.1 \pm 5.40$, compared to the SNR of $8.4 \pm 2.19$ obtained by the surface coil (Figure 5). In Figure 6, as expected, the average SNR in ROIs obtained using the MRIG were $16.9,15.2$, and 15.5 , which was much higher than the SNR of 6.2, 7.3, and 6.6, obtained using the surface coil.

The HE staining in Figure 7 showed no evidence of injury at the target vessel walls.

\section{Discussion}

It is difficult to build an MRIG inserted into a vessel smoothly without scratching the vessel wall and causing spasms. To achieve this, the tip of the MRIG needs to be soft while maintaining a certain degree of toughness. To this end, we stripped off the outer conductor of a commercial coaxial cable but maintained its insulating layer. This insulating layer wrapped around the inner conductor to sufficiently maintain its mechanical properties.

In conclusion, we validated the feasibility of safely generating $0.2 \mathrm{~mm} \times 0.2 \mathrm{~mm} \times 2 \mathrm{~mm} \mathrm{MR}$ images of the coronary artery wall in rich detail using a 0.014 -inch MRIG. The technique helps to lay the foundation of clinical practice for high-resolution MRI of coronary artery plaques. 


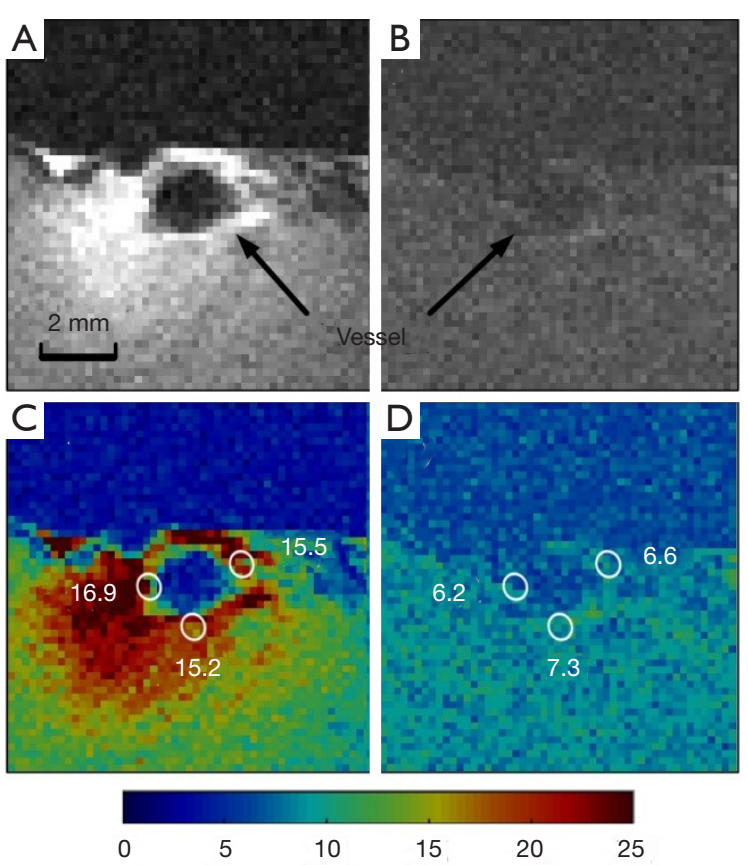

Figure 6 The anatomies (A, B) and SNR maps (C, D) of the LAD branch were obtained by high-resolution imaging using MRIG (A, C) and commercial surface coils (B, D). The selected ROIs show that the SNRs of the MRIG in the vicinity of the vessel wall are higher than those of the surface coil. SNR, signal-to-noise; LAD, left anterior descending; MRIG, magnetic resonance imaging guidewire.
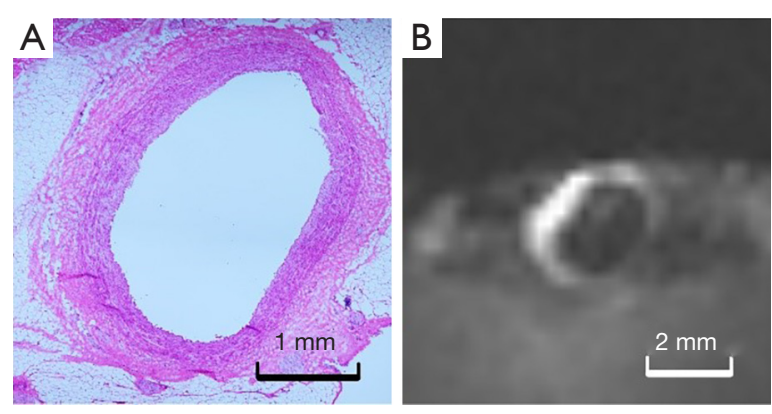

Figure 7 The pathological section and MRI of a vessel. (A) The pathological section showed no evidence of injury. (B) The MRI by MRIG at the resolution of $0.2 \mathrm{~mm} \times 0.2 \mathrm{~mm} \times 2 \mathrm{~mm}$. MRIG, magnetic resonance imaging guidewire.

\section{Acknowledgments}

Funding: This work was supported by the National Natural Science Foundation of China (Grant No.: 81671789); the Project of Health Commission of Shanxi Province
(No.: 2018109); National Key R\&D Program of China 2021YFE0204400; the Strategic Priority Research Program of the Chinese Academy of Sciences (Grant No. XDB25000000); city grant RCYX20200714114735123; Guangdong Province grant 2020B1212060051; and Youth Innovation Promotion Association of CAS No. 2017415.

\section{Footnote}

Conflicts of Interest: All authors have completed the ICMJE uniform disclosure form (available at https:// dx.doi.org/10.21037/qims-21-286). Dr. XZ serves as an Associate Editor of Quantitative Imaging in Medicine and Surgery. Dr. YM reports that his institution is funded by the National Natural Science Foundation of China (Grant No.: 81671789) and the Project of Health Commission of Shanxi Province (No.: 2018109). Dr. YL reports that his institution is funded by the National Key R\&D Program of China 2021YFE0204400; the Strategic Priority Research Program of the Chinese Academy of Sciences (Grant No. XDB25000000); city grant RCYX20200714114735123; Guangdong Province grant 2020B1212060051; and Youth Innovation Promotion Association of CAS No. 2017415. The other authors have no conflicts of interest to declare.

Ethical Statement: The authors are accountable for all aspects of the work by ensuring that questions related to the accuracy or integrity of any part of the work are appropriately investigated and resolved. Ethical approval and written informed consent were not applicable because no human subjects were involved in our experiments.

Open Access Statement: This is an Open Access article distributed in accordance with the Creative Commons Attribution-NonCommercial-NoDerivs 4.0 International License (CC BY-NC-ND 4.0), which permits the noncommercial replication and distribution of the article with the strict proviso that no changes or edits are made and the original work is properly cited (including links to both the formal publication through the relevant DOI and the license). See: https://creativecommons.org/licenses/by-nc-nd/4.0/.

\section{References}

1. Virani SS, Alonso A, Benjamin EJ, Bittencourt MS, Callaway CW, Carson AP, et al. Heart Disease and Stroke Statistics-2020 Update: A Report From the American Heart Association. Circulation 2020;141:e139-596. 
2. Gheorghe A, Griffiths U, Murphy A, Legido-Quigley H, Lamptey P, Perel P. The economic burden of cardiovascular disease and hypertension in low- and middle-income countries: a systematic review. BMC Public Health 2018;18:975.

3. Douglas G, Channon KM. The pathogenesis of atherosclerosis. Medicine 2014;42:480-4.

4. Finn AV, Nakano M, Narula J, Kolodgie FD, Virmani R. Concept of vulnerable/unstable plaque. Arterioscler Thromb Vasc Biol 2010;30:1282-92.

5. Saba L, Yuan C, Hatsukami TS, Balu N, Qiao Y, DeMarco JK, Saam T, Moody AR, Li D, Matouk CC, Johnson MH, Jäger HR, Mossa-Basha M, Kooi ME, Fan Z, Saloner D, Wintermark M, Mikulis DJ, Wasserman BA; Vessel Wall Imaging Study Group of the American Society of Neuroradiology. Carotid Artery Wall Imaging: Perspective and Guidelines from the ASNR Vessel Wall Imaging Study Group and Expert Consensus Recommendations of the American Society of Neuroradiology. AJNR Am J Neuroradiol 2018;39:E9-E31.

6. Wei H, Zhang M, Li Y, Zhao X, Canton G, Sun J, Xu D, Zhou Z, Chen S, Ferguson MS, Hatsukami TS, Li R, Yuan C. Evaluation of 3D multi-contrast carotid vessel wall MRI: a comparative study. Quant Imaging Med Surg 2020;10:269-82.

7. Zhu T, Ren L, Zhang L, Shao Y, Wan L, Li Y, Liang D, Zheng H, Liu X, Zhang N. Comparison of plaque characteristics of small and large subcortical infarctions in the middle cerebral artery territory using high-resolution magnetic resonance vessel wall imaging. Quant Imaging Med Surg 2021;11:57-66.

8. Hu X, Zhang L, Zhang X, Zhu H, Chen X, Zhang Y, Chung YC, Liu X, Zheng H, Li Y. An 8-channel RF coil array for carotid artery MR imaging in humans at $3 \mathrm{~T}$. Med Phys 2016;43:1897.

9. Hu X, Li Y, Zhang L, Zhang X, Liu X, Chung YC. A 32-channel coil system for MR vessel wall imaging of intracranial and extracranial arteries at 3T. Magn Reson Imaging 2017;36:86-92.

10. Li Y, Chen Q, Wei Z, Zhang L, Tie C, Zhu Y, Jia S, Xia J, Liang D, He Q, Zhang X, Liu X, Zhang B, Zheng H. One-Stop MR Neurovascular Vessel Wall Imaging With a 48-Channel Coil System at 3 T. IEEE Trans Biomed Eng 2020;67:2317-27.

11. Mandell DM, Mossa-Basha M, Qiao Y, Hess CP, Hui F, Matouk C, Johnson MH, Daemen MJ, Vossough A, Edjlali M, Saloner D, Ansari SA, Wasserman BA, Mikulis DJ; Vessel Wall Imaging Study Group of the American
Society of Neuroradiology. Intracranial Vessel Wall MRI: Principles and Expert Consensus Recommendations of the American Society of Neuroradiology. AJNR Am J Neuroradiol 2017;38:218-29.

12. Abd-Elmoniem KZ, Gharib AM, Pettigrew RI. Coronary vessel wall 3-T MR imaging with time-resolved acquisition of phase-sensitive dual inversion-recovery (TRAPD) technique: initial results in patients with risk factors for coronary artery disease. Radiology 2012;265:715-23.

13. Botnar RM, Stuber M, Lamerichs R, Smink J, Fischer SE, Harvey P, Manning WJ. Initial experiences with in vivo right coronary artery human $\mathrm{MR}$ vessel wall imaging at 3 tesla. J Cardiovasc Magn Reson 2003;5:589-94.

14. Gutierrez J, Elkind MS, Petito C, Chung DY, Dwork AJ, Marshall RS. The contribution of HIV infection to intracranial arterial remodeling: a pilot study. Neuropathology 2013;33:256-63.

15. Martin AJ, Henkelman RM. Intravascular MR imaging in a porcine animal model. Magn Reson Med 1994;32:224-9.

16. Quick HH, Ladd ME, Zimmermann-Paul GG, Erhart P, Hofmann E, von Schulthess GK, Debatin JF. Singleloop coil concepts for intravascular magnetic resonance imaging. Magn Reson Med 1999;41:751-8.

17. Zhang X, Martin A, Jordan C, Lillaney P, Losey A, Pang Y, $\mathrm{Hu}$ J, Wilson M, Cooke D, Hetts SW. Design of catheter radio frequency coils using coaxial transmission line resonators for interventional neurovascular MR imaging. Quant Imaging Med Surg 2017;7:187-94.

18. Jordan CD, Thorne BRH, Wadhwa A, Losey AD, Ozhinsky E, Kondapavulur S, Fratello V, Moore T, Stillson C, Yee C, Watkins RD, Scott GC, Martin AJ, Zhang X, Wilson MW, Hetts SW. Wireless Resonant Circuits Printed Using Aerosol Jet Deposition for MRI Catheter Tracking. IEEE Trans Biomed Eng 2020;67:876-82.

19. Ocali O, Atalar E. Intravascular magnetic resonance imaging using a loopless catheter antenna. Magn Reson Med 1997;37:112-8.

20. Yang X, Atalar E. Intravascular MR imaging-guided balloon angioplasty with an MR imaging guide wire: feasibility study in rabbits. Radiology 2000;217:501-6.

21. Qiu B, Karmarkar P, Brushett C, Gao F, Kon R, Kar S, Atalar E, Yang X. Development of a 0.014-inch magnetic resonance imaging guidewire. Magn Reson Med 2005;53:986-90.

22. Qiu B, Gao F, Karmarkar P, Atalar E, Yang X. Intracoronary MR imaging using a 0.014-inch MR imaging-guidewire: toward MRI-guided coronary interventions. J Magn Reson Imaging 2008;28:515-8. 
23. Qian D, Bottomley PA. High-resolution intravascular magnetic resonance quantification of atherosclerotic plaque at 3T. J Cardiovasc Magn Reson 2012;14:20.

24. Wu B, Zhang X, Wang C, Li Y, Pang Y, Lu J, Xu D, Majumdar S, Nelson SJ, Vigneron DB. Flexible transceiver array for ultrahigh field human MR imaging. Magn Reson Med 2012;68:1332-8.

25. Li Y, Wang C, Yu B, Vigneron D, Chen W, Zhang X. Image homogenization using pre-emphasis method for high field MRI. Quant Imaging Med Surg 2013;3:217-23.

26. Zhang X, Ji JX. Parallel and sparse MR imaging: methods and instruments-Part 1. Quant Imaging Med Surg 2014;4:1-3.

27. Pang Y, Wong EW, Yu B, Zhang X. Design and numerical evaluation of a volume coil array for parallel MR imaging at ultrahigh fields. Quant Imaging Med Surg 2014;4:50-6.

28. Hu X, Chen X, Liu X, Zheng H, Li Y, Zhang X. Parallel imaging performance investigation of an 8-channel common-mode differential-mode (CMDM) planar array for 7T MRI. Quant Imaging Med Surg 2014;4:33-42.

Cite this article as: Meng Y, Mo Z, Hao J, Peng Y, Yan H, Mu J, Ma D, Zhang X, Li Y. High-resolution intravascular magnetic resonance imaging of the coronary artery wall at 3.0 Tesla: toward evaluation of atherosclerotic plaque vulnerability. Quant Imaging Med Surg 2021;11(11):4522-4529. doi: 10.21037/qims-21286
29. Yan X, Xue R, Zhang X. A monopole/loop dual-tuned RF coil for ultrahigh field MRI. Quant Imaging Med Surg 2014;4:225-31.

30. Pang Y, Wu B, Jiang X, Vigneron DB, Zhang X. Tilted microstrip phased arrays with improved electromagnetic decoupling for ultrahigh-field magnetic resonance imaging. Medicine (Baltimore) 2014;93:e311.

31. Hofmann LV, Sood S, Liddell RP, Gupta A, Arepally A, Rodriguez ER, Eng J. Arteriographic and pathologic evaluation of two suture-mediated arterial closure devices in a porcine model. J Vasc Interv Radiol 2003;14:755-61.

32. Susil RC, Yeung CJ, Atalar E. Intravascular extended sensitivity (IVES) MRI antennas. Magn Reson Med 2003;50:383-90.

33. Keil B, Wald LL. Massively parallel MRI detector arrays. J Magn Reson 2013;229:75-89.

34. Meng Y, Zhang F, Gu H, Wang J, Yuan C, Zhang Z, Qiu $\mathrm{B}$, Yang X. Intravascular 3.0 T MRI using an imagingguidewire: a feasibility study in swine. Appl Magn Reson 2011;40:105-112. 